\title{
On the space of quantum fields in massive two-dimensional theories
}

\author{
Gesualdo Delfino \\ International School for Advanced Studies (SISSA) \\ via Beirut 2-4, 34014 Trieste, Italy \\ INFN sezione di Trieste
}

\begin{abstract}
For a large class of integrable quantum field theories we show that the $S$-matrix determines a space of fields which decomposes into subspaces labeled, besides the charge and spin indices, by an integer $k$. For scalar fields $k$ is non-negative and is naturally identified as an off-critical extension of the conformal level. To each particle we associate an operator acting in the space of fields whose eigenvectors are primary $(k=0)$ fields of the massive theory. We discuss how the existing results for models as different as $Z_{n}$, sine-Gordon or Ising with magnetic field fit into this classification.
\end{abstract}




\section{Introduction}

The classification of quantum fields according to the scaling dimension determined by the short distance behavior of correlation functions is at the basis of the renormalization group idea and its many applications to physics. The field theoretical description of critical phenomena assumes the existence of a spectrum of scaling dimensions bounded from below, with the lowest dimension (primary) fields determining the critical exponents and the others responsible for corrections to scaling. Such a scheme is expected to hold in the generic case of non-trivial fixed points and also in absence of reflection positivity, when the scaling dimensions are not necessarily positive.

An exact confirmation exists for the fixed point (conformal) field theories in two dimensions [1. There, remarkably, the semi-infinite spectrum of scaling dimensions emerges in connection with the lowest weight representations of the infinite-dimensional conformal group. It is found that each representation corresponds to a family of fields whose scaling dimensions differ by integers. For scalar fields this gradation according to the dimension can be made in terms of a single non-negative integer called level.

Two-dimensional quantum field theory allows also for massive exactly solvable (integrable) theories [2]. Here, however, the solution comes in the form of an exact $S$-matrix, and the characterization of the space of fields starting only from particle dynamics, important in principle as well as for applications to off-critical systems, is a non-trivial task. A set of functional equations [3, 4] is known for the matrix elements of fields on particle states (form factors). The space of fields of the massive theory corresponds to the space of solutions of the form factor equations. These, however, contain only the symmetry data (charges and spin) of the field, and nothing about its scaling dimension. With time, evidence of a relation between the high energy asymptotics of form factors and the conformal levels emerged. The first result in this sense was obtained in [5], but only recently the isomorphism between critical and off-critical field spaces has been shown for the simplest massive model originating from a non-trivial fixed point of the renormalization group [6].

The emergence of a sufficiently general pattern, however, had been so far prevented by the difficulty of controlling the role of model dependent features such as the particle spectrum, the symmetries and the properties of mutual locality between particles and fields. In this paper we show that the space of fields admits an unifying characterization within a large class of integrable theories, those with reflectionless scattering at high energies and additive charges. Starting from the $S$-matrix, the space of fields can be decomposed into subspaces labeled by four indices. Three of them encode the internal and Lorentz symmetry properties of the field, while the fourth is an integer, let us call it $k$, related to the asymptotic behavior of form factors. We obtain a lower bound for the asymptotic behavior which amounts to a lower bound on $k$. For scalar fields $k$ is non-negative and is naturally identified as playing the same role as the level in the conformal classification. We also associate to each particle species $a$ an operator $\Lambda_{a}$ mapping fields into fields and whose "eigenvectors" are primary $(k=0)$ fields. We show how this scheme applies to models $\left(\mathbf{Z}_{N}\right.$, sine-Gordon, the theories without internal symmetries) 
which are usually seen as sharing little else than integrability.

The paper is organized as follows. In the next section we recall the features of factorized scattering theories and, after restricting to the subclass of interest in this paper, we give a characterization of the associated space of fields in terms of spin and charges. In section 3 we introduce the operators $\Lambda_{a}$, further classify the fields according to the asymptotic behavior of form factors and discuss the analogies with the conformal classification. In section 4 we specialize the discussion to several models before making few concluding remarks in section 5 .

\section{Integrable quantum field theories}

Integrable quantum field theories are characterized by a completely elastic and factorized $S$ matrix [2]. If we denote by $A_{a}(\theta)$ a particle of species $a$, with mass $m_{a}$ and energy-momentum $\left(p^{0}, p^{1}\right)=\left(m_{a} \cosh \theta, m_{a} \sinh \theta\right)$, the $S$-matrix is completely determined by the two-particle scattering amplitudes associated to the processes

$$
\left|A_{a}\left(\theta_{1}\right) A_{b}\left(\theta_{2}\right)\right\rangle_{\text {in }}=S_{a b}^{c d}\left(\theta_{1}-\theta_{2}\right)\left|A_{c}\left(\theta_{1}\right) A_{d}\left(\theta_{2}\right)\right\rangle_{\text {out }} .
$$

The theories we consider are invariant under charge conjugation, space and time reversal, so that the amplitudes satisfy

$$
\begin{aligned}
& S_{a b}^{c d}(\theta)=S_{\bar{a} \bar{b}}^{\bar{c} \bar{d}}(\theta), \\
& S_{a b}^{c d}(\theta)=S_{b a}^{d c}(\theta), \\
& S_{a b}^{c d}(\theta)=S_{c d}^{a b}(\theta),
\end{aligned}
$$

where indices with a bar denote anti-particles.

The scattering amplitudes satisfy the unitarity, crossing, bootstrap and factorization equations

$$
\begin{gathered}
S_{a b}^{e f}(\theta) S_{e f}^{c d}(-\theta)=\delta_{a}^{c} \delta_{b}^{d}, \\
S_{a b}^{c d}(i \pi-\theta)=S_{a \bar{d}}^{c \bar{b}}(\theta), \\
\Gamma_{a b}^{c} S_{d c}^{e f}(\theta)=\Gamma_{h j}^{f} S_{d a}^{i h}\left(\theta-i \bar{u}_{a \bar{c}}^{\bar{b}}\right) S_{i b}^{e j}\left(\theta+i \bar{u}_{b \bar{c}}^{\bar{a}}\right), \\
S_{a b}^{d e}\left(\theta_{1}-\theta_{2}\right) S_{d c}^{f g}\left(\theta_{1}-\theta_{3}\right) S_{e g}^{h i}\left(\theta_{2}-\theta_{3}\right)=S_{b c}^{d e}\left(\theta_{2}-\theta_{3}\right) S_{a e}^{g i}\left(\theta_{1}-\theta_{3}\right) S_{g d}^{f h}\left(\theta_{1}-\theta_{2}\right),
\end{gathered}
$$

where repeated indices are summed over, $i u_{a b}^{c}$ is the resonant rapidity difference associated to the bound state formation $A_{a} A_{b} \rightarrow A_{c}, \bar{u}_{a b}^{c} \equiv \pi-u_{a b}^{c}$, and the three particle couplings $\Gamma_{a b}^{c}$ are determined by

$$
\operatorname{Res}_{\theta=i u_{a b}^{c}} S_{a b}^{d e}(\theta)=i \Gamma_{a b}^{c} \Gamma_{\bar{d} \bar{e}}^{\bar{c}} .
$$

The above equations are normally sufficient for the exact determination of the scattering amplitudes.

In this paper we consider integrable theories invariant under the action of an abelian group of transformations $G$, whose $S$-matrix becomes diagonal at high energies, i.e. satisfies

$$
\lim _{\theta \rightarrow \pm \infty} S_{a b}^{c d}(\theta)=e^{ \pm 2 i \pi \alpha_{a b}} \delta_{a}^{c} \delta_{b}^{d} ;
$$


to be definite, in the following we take $\alpha_{a b} \in[0,1)$.

In the integrable quantum field theories the fields $\Phi(x)$ are constructed from the knowledge of the $S$-matrix through the determination of the form factors

$$
F_{a_{1} \ldots a_{n}}^{\Phi}\left(\theta_{1}, \ldots, \theta_{n}\right)=\left\langle 0|\Phi(0)| A_{a_{1}}\left(\theta_{1}\right) \ldots A_{a_{n}}\left(\theta_{n}\right)\right\rangle,
$$

where $|0\rangle$ denotes the vacuum (i.e. zero-particle) state. If $C_{a}$ and $C_{\Phi}$ denote the charges of $A_{a}$ and $\Phi$ with respect to the group $G$, the form factors (2.11) vanish by symmetry unless

$$
\sum_{j=1}^{n} C_{a_{j}}=-C_{\Phi} .
$$

The form factors satisfy the equations [3, 4]

$$
\begin{aligned}
& F_{a_{1} \ldots a_{n}}^{\Phi}\left(\theta_{1}+\Lambda, \ldots, \theta_{n}+\Lambda\right)=e^{s_{\Phi} \Lambda} F_{a_{1} \ldots a_{n}}^{\Phi}\left(\theta_{1}, \ldots, \theta_{n}\right) \\
& F_{\ldots a_{i} a_{i+1} \ldots}^{\Phi}\left(\ldots, \theta_{i}, \theta_{i+1}, \ldots\right)=S_{a_{i} a_{i+1}}^{b_{i} b_{i+1}}\left(\theta_{i}-\theta_{i+1}\right) F_{\ldots b_{i+1} b_{i} \ldots}^{\Phi}\left(\ldots, \theta_{i+1}, \theta_{i}, \ldots\right), \\
& F_{a_{1} \ldots a_{n}}^{\Phi}\left(\theta_{1}+2 i \pi, \theta_{2}, \ldots, \theta_{n}\right)=e^{-2 i \pi \gamma_{\Phi, a_{1}}} F_{a_{2} \ldots a_{n} a_{1}}^{\Phi}\left(\theta_{2}, \ldots, \theta_{n}, \theta_{1}\right), \\
& \operatorname{Res}_{\theta_{a}-\theta_{b}=i u_{a b}^{c}} F_{a b a_{1} \ldots a_{n}}^{\Phi}\left(\theta_{a}, \theta_{b}, \theta_{1}, \ldots, \theta_{n}\right)=i \Gamma_{a b}^{c} F_{c a_{1} \ldots a_{n}}^{\Phi}\left(\theta_{c}, \theta_{1}, \ldots, \theta_{n}\right), \\
& \operatorname{Res}_{\theta^{\prime}=\theta+i \pi} F_{\bar{a} a a_{1} \ldots a_{n}}^{\Phi}\left(\theta^{\prime}, \theta, \theta_{1}, \ldots, \theta_{n}\right)= \\
& \quad i\left[\delta_{a_{1}}^{b_{1}} \ldots \delta_{a_{n}}^{b_{n}}-e^{2 i \pi \gamma_{\Phi, a}} S_{a_{1} \ldots a_{n}}^{b_{1} \ldots b_{n}}\left(\theta \mid \theta_{1}, \ldots, \theta_{n}\right)\right] F_{b_{1} \ldots b_{n}}^{\Phi}\left(\theta_{1}, \ldots, \theta_{n}\right),
\end{aligned}
$$

where $s_{\Phi}$ is the euclidean spin of the field $\Phi(x)$,

$$
S_{a_{1} \ldots a_{n}}^{b_{1} \ldots b_{n}}\left(\theta \mid \theta_{1}, \ldots, \theta_{n}\right) \equiv S_{a_{1} \alpha_{n}}^{b_{1} \alpha_{1}}\left(\theta-\theta_{1}\right) S_{a_{2} \alpha_{1}}^{b_{2} \alpha_{2}}\left(\theta-\theta_{2}\right) \ldots S_{a_{n} \alpha_{n-1}}^{b_{n} \alpha_{n}}\left(\theta-\theta_{n}\right)
$$

and a non-integer $\gamma_{\Phi, a}$ accounts for a semi-locality between the field $\Phi$ and the particle $A_{a}$ (see e.g. [7]). If $z=x_{1}+i x_{2}$ and $\bar{z}=x_{1}-i x_{2}$ are complex coordinates on the plane, we say that two fields $\Phi_{1}$ and $\Phi_{2}$ are mutually semi-local with semi-locality index $\gamma_{\Phi_{1}, \Phi_{2}}$ if

$$
\left\langle\cdots \Phi_{1}\left(z e^{2 i \pi}, \bar{z}^{-2 i \pi}\right) \Phi_{2}(0) \cdots\right\rangle=e^{2 i \pi \gamma_{\Phi_{1}}, \Phi_{2}}\left\langle\cdots \Phi_{1}(z, \bar{z}) \Phi_{2}(0) \cdots\right\rangle .
$$

Clearly, $\gamma_{\Phi_{1}, \Phi_{2}}$ is defined up to integers, and in the following we take it in the interval $[0,1)$. Denoting $\varphi_{a}(x)$ a field which interpolates the particle $A_{a}$, i.e. a field with

$$
\left\langle A_{a}(\theta)\left|\varphi_{a}(x)\right| 0\right\rangle \neq 0,
$$

we have

$$
\gamma_{\Phi, a}=\gamma_{\Phi, \varphi_{a}} .
$$

When writing (2.15) and (2.17) and throughout this paper we choose, without loss of generality, the fields which interpolate the particles to be spinless.

Since the form factors (2.11) determine by crossing all the matrix elements of $\Phi$, and since the knowledge of all the matrix elements completely determines the field, the space of solutions 
of the equations (2.13)-(2.17) determines the space of fields of the theory1, which we denote by $\mathcal{F}$. Clearly, $\mathcal{F}$ can be decomposed into subspaces containing fields with the same charge, spin and semi-locality indices, which are the field data entering the form factor equations. These subspaces are always infinite-dimensional.

This decomposition is conveniently characterized in the following way. The semi-locality property (2.19) can be accounted for introducing a second additive quantity $\tilde{C}$, that we call dual charge, in such a way that we can write

$$
\mathcal{F}=\bigoplus_{C, \tilde{C}} \mathcal{F}_{C, \tilde{C}}
$$

where $\mathcal{F}_{C, \tilde{C}}$ is the subspace containing the fields with charge $C$ and dual charge $\tilde{C}$. The semilocality index entering (2.19) is given by

$$
\gamma_{\Phi_{1}, \Phi_{2}}=\kappa\left(C_{\Phi_{1}} \tilde{C}_{\Phi_{2}}+\tilde{C}_{\Phi_{1}} C_{\Phi_{2}}\right)
$$

with $\kappa$ a field-independent normalization for the dual charge that we introduce for convenience. Additivity of the charges ensures the property $\gamma_{\Phi_{1} \Phi_{2}, \Phi_{3}}=\gamma_{\Phi_{1}, \Phi_{3}}+\gamma_{\Phi_{2}, \Phi_{3}}$. We stipulate that the quanta of the charge $C$ are measured in integer units, so that $\kappa \tilde{C}$ is defined modulo integers like the semi-locality indices.

The energy-momentum tensor $T^{\mu \nu}$ is neutral and local with respect to all the fields in the theory, and belongs to $\mathcal{F}_{0,0}$. We will call "order" fields the fields belonging to $\mathcal{F}_{C, 0}$ with $C \neq 0$, and "disorder" fields the fields belonging to $\mathcal{F}_{0, \tilde{C}}$ with $\tilde{C} \neq 0$. Order and disorder fields are mutually non-local.

If $\Phi_{3}$ is produced in the operator product expansion of $\Phi_{1}$ and $\Phi_{2}$, (2.19) leads to the relation $\gamma_{\Phi_{1}, \Phi_{2}}=s_{\Phi_{3}}-s_{\Phi_{1}}-s_{\Phi_{2}}$. Denote by $\bar{\Phi}$ the charge and dual charge conjugate of $\Phi$ $\left(C_{\Phi}+C_{\bar{\Phi}}=\tilde{C}_{\Phi}+\tilde{C}_{\bar{\Phi}}=0\right) ; \Phi$ and $\bar{\Phi}$ have the same spin and their operator product expansion produces only neutral fields, the identity among them. Then we have $\gamma_{\Phi, \bar{\Phi}}=-2 s_{\Phi}$, so that comparison with (2.23) gives

$$
s_{\Phi}=\kappa C_{\Phi} \tilde{C}_{\Phi}+n_{\Phi}, \quad n_{\Phi} \in \mathbf{Z}
$$

with $n_{\Phi}$ accounting for the fact that the semi-locality indices are defined up to integers. If $s_{\Phi}$ is non-integer (this requires $C_{\Phi}$ and $\tilde{C}_{\Phi}$ both non-zero), $\Phi$ is said to be a parafermionic field [8] , as a generalization of the fermionic fields corresponding to half-integer spin. The field subspaces $\mathcal{F}_{C, \tilde{C}}$ can further be decomposed according to the spin in the form

$$
\mathcal{F}_{C, \tilde{C}}=\bigoplus_{n \in \mathbf{Z}} \mathcal{F}_{C, \tilde{C}}^{n},
$$

the superscript $n$ being the integer in (2.24). For $\mathcal{F}_{0,0} n$ is the spin itself. The subspaces $\mathcal{F}_{C, \tilde{C}}^{n}$ contain fields corresponding to solutions of the form factor equations (2.13) $-(2.17)$ with the same values of $s_{\Phi}, C_{\Phi}$ and $\gamma_{\Phi, a}$, and are the above mentioned infinite-dimensional subspaces.

\footnotetext{
${ }^{1}$ In some cases the theory allows also for fields which are not simply semi-local with respect to the particles and require a modification of (2.15), 2.17). In these cases $\mathcal{F}$, as defined above, is not the full space of fields. It is, however, a subspace closed under operator product expansion.
} 


\section{The operators $\Lambda_{a}$}

Condition (2.10), together with (2.14) and (2.15), yields

$$
\begin{gathered}
\lim _{\theta_{n} \rightarrow+\infty} F_{a_{1} \ldots a_{n}}^{\Phi}\left(\theta_{1}, \ldots, \theta_{n-1}, \theta_{n}+2 i \pi\right)=\prod_{j=1}^{n-1} e^{-2 i \pi \alpha_{a_{j} a_{n}}} \lim _{\theta_{n} \rightarrow+\infty} F_{a_{n} a_{1} \ldots a_{n-1}}^{\Phi}\left(\theta_{n}+2 i \pi, \theta_{1}, \ldots, \theta_{n-1}\right) \\
=e^{-2 i \pi \gamma_{\Phi, a_{n}}} \prod_{j=1}^{n-1} e^{-2 i \pi \alpha_{a_{j} a_{n}}} \lim _{\theta_{n} \rightarrow+\infty} F_{a_{1} \ldots a_{n}}^{\Phi}\left(\theta_{1}, \ldots, \theta_{n}\right),
\end{gathered}
$$

and then

$$
F_{a_{1} \ldots a_{n}}^{\Phi}\left(\theta_{1}, \ldots, \theta_{n}\right)=f_{a_{1} \ldots a_{n-1}}^{\Phi, a_{n}}\left(\theta_{1}, \ldots, \theta_{n-1}\right) e^{y_{\Phi, a_{n}} \theta_{n}}, \quad \theta_{n} \rightarrow+\infty, \quad n>1
$$

with

$$
y_{\Phi, a_{n}}=-\gamma_{\Phi, a_{n}}-\sum_{j=1}^{n-1} \alpha_{a_{j} a_{n}}+n_{\Phi, a_{n}}, \quad n_{\Phi, a_{n}} \in \mathbf{Z} .
$$

In writing (3.3) we imply that, for $n>1$, the r.h.s. depends on $\Phi$ and $a_{n}$ only, a property that can be shown as follows. We can change the particle state while preserving (2.12) in two ways: i) we add/remove a particle-antiparticle pair $A_{a} A_{\bar{a}}$. In this case the product in (3.1) acquires/loses a factor

$$
\lim _{\theta \rightarrow-\infty} S_{a a_{n}}^{a a_{n}}(\theta) S_{\bar{a} a_{n}}^{\bar{a} a_{n}}(\theta)=\lim _{\theta \rightarrow-\infty} S_{a a_{n}}^{a a_{n}}(\theta) S_{a a_{n}}^{a a a_{n}}(i \pi-\theta)=1,
$$

where (2.10) and (2.6) have been used;

ii) if $\Gamma_{a b}^{c} \neq 0$, we trade $A_{c}$ for $A_{a} A_{b}$, or vice versa. In this case the product in (3.1) acquires/loses a factor

$$
\lim _{\theta \rightarrow-\infty} S_{a a_{n}}^{a a_{n}}(\theta) S_{b a_{n}}^{b a_{n}}(\theta) / S_{c a_{n}}^{c a_{n}}(\theta)=1,
$$

where (2.10) and (2.7) have been used.

This shows that the sum in (3.3) depends on $\Phi$ and $a_{n}$ only. Finally, also the integer $n_{\Phi, a_{n}}$ must have this property in such a way that the two sides of (2.16) and (2.17), which relate matrix elements with a different number of particles, have the same limit as $\theta_{n} \rightarrow+\infty$.

The case $n=1$ cannot be included in (3.2) also because it follows from (2.13) that the asymptotic limit of $F_{a}^{\Phi}(\theta)$ is determined by the spin $s_{\Phi}$, which in general does not coincide with $y_{\Phi, a}$. We now extend (3.2) to the form

$$
\lim _{\theta \rightarrow+\infty} e^{-y_{\Phi, a} \theta} F_{a_{1} \ldots a_{n} a}^{\Phi}\left(\theta_{1}, \ldots, \theta_{n}, \theta\right)=f_{a_{1} \ldots a_{n}}^{\Phi, a}\left(\theta_{1}, \ldots, \theta_{n}\right), \quad n \geq 0
$$

which for $n=0$ associates to $F_{a}^{\Phi}$ the constant $f^{\Phi, a}$. With this additional definition we can multiply both sides of (2.13)-(2.17) by $e^{-y_{\Phi, a_{n}} \theta_{n}}$ and take the limit $\theta_{n} \rightarrow+\infty$, for any number of particles. The result of this operation is that the functions $f_{a_{1} \ldots a_{n-1}}^{\Phi, a_{n}}\left(\theta_{1}, \ldots, \theta_{n-1}\right)$ with $n \geq 1$, 
satisfy the equations corresponding to the form factors $F_{a_{1} \ldots a_{n-1}}^{\Phi_{\left(a_{n}\right)}}\left(\theta_{1}, \ldots, \theta_{n-1}\right)$, where $\Phi_{(a)}$ is a field with spin, semi-locality indices and charge given by

$$
\begin{aligned}
& s_{\Phi_{(a)}}=s_{\Phi}-y_{\Phi, a}, \\
& \gamma_{\Phi_{(a)}, b}=\gamma_{\Phi, b}-\alpha_{a b}, \\
& C_{\Phi_{(a)}}=C_{\Phi}+C_{a} .
\end{aligned}
$$

In other words, the limiting procedure (3.6) defines an operation in the space of fields which maps $\Phi$ into $\Phi_{(a)}$. Denoting by $\Lambda_{a}$ the corresponding operator, we can write

$$
\Lambda_{a} \Phi=\Phi_{(a)},
$$

or, on matrix elements,

$$
\begin{aligned}
\Lambda_{a} F_{a_{1} \ldots a_{n} a}^{\Phi}\left(\theta_{1}, \ldots, \theta_{n}, \theta\right) & =\lim _{\theta \rightarrow+\infty} e^{-y_{\Phi, a} \theta} F_{a_{1} \ldots a_{n} a}^{\Phi}\left(\theta_{1}, \ldots, \theta_{n}, \theta\right) \\
& =F_{a_{1} \ldots a_{n}}^{\Phi_{(a)}}\left(\theta_{1}, \ldots, \theta_{n}\right), \quad n \geq 0
\end{aligned}
$$

The action of $\Lambda_{a}$ in (3.11) yields, by construction, a finite non-zero result for $n>0$. For $n=0,(2.13)$ and (3.7) give

$$
\left\langle 0\left|\Phi_{(a)}(0)\right| 0\right\rangle=\Lambda_{a} F_{a}^{\Phi}(\theta)=F_{a}^{\Phi}(0) \lim _{\theta \rightarrow+\infty} e^{s_{\Phi}(a)} \theta .
$$

The requirement that all fields in the theory have finite vacuum expectation value implies $s_{\Phi_{(a)}} \leq$ 0 if $F_{a}^{\Phi}(\theta) \neq 0$, i.e.

$$
y_{\Phi, a} \geq s_{\Phi} \quad \text { if } \quad F_{a}^{\Phi}(\theta) \neq 0 .
$$

The vanishing of (3.12) for $s_{\Phi_{(a)}}<0$ is in agreement with Lorentz invariance, which prescribes that only spinless fields can have a non-zero vacuum expectation value.

Notice that if

$$
\Phi(x)=\sum_{k=1}^{K} c_{k} \Phi_{k}(x)
$$

we obtain

$$
\Lambda_{a} \Phi=\left\{\begin{array}{rlc}
c_{1} \Lambda_{a} \Phi_{1} & \text { if } & y_{\Phi_{1}}>y_{\Phi_{2}}>\ldots>y_{\Phi_{K}} \\
\sum_{k=1}^{K} c_{k} \Lambda_{a} \Phi_{k} & \text { if } & y_{\Phi_{1}}=y_{\Phi_{2}}=\ldots=y_{\Phi_{K}} .
\end{array}\right.
$$

Using the notations

$$
\Theta \equiv T_{\mu}^{\mu}, \quad \Theta_{a} \equiv \Theta_{(a)}=\Lambda_{a} \Theta
$$

(3.7) $-(3.9)$ give

$$
s_{\Theta_{a}}=-y_{\Theta, a}, \quad \gamma_{\Theta_{a}, b}=-\alpha_{a b}, \quad C_{\Theta_{a}}=C_{a},
$$

where we also used the fact that the trace of the energy-momentum tensor is a spinless field in the Lorentz invariant theories we consider. 
The spinless field $\varphi_{a}$ we have chosen to interpolate the particle $A_{a}$ belongs to $\mathcal{F}_{C_{a}, 0}^{0}$. Hence, (3.17) and (2.23) give

$$
\alpha_{a b}=-\kappa C_{a} \tilde{C}_{\Theta_{b}}=-\kappa \tilde{C}_{\Theta_{a}} C_{b},
$$

the last equality following from the symmetry of $\alpha_{a b}$ in the two indices. As a consequence, (3.8) takes the form $\gamma_{\Phi_{(a)}, b}=\kappa\left(\tilde{C}_{\Phi}+\tilde{C}_{\Theta_{a}}\right) C_{b}$, so that we have

$$
\tilde{C}_{\Phi_{(a)}}=\tilde{C}_{\Phi}+\tilde{C}_{\Theta_{a}}(\bmod \mathbf{Z} / \kappa) .
$$

Recalling also (3.3) and (3.7) we obtain

$$
\begin{aligned}
& y_{\Phi, a}=s_{\Phi}-s_{\Phi(a)}=-\kappa\left[C_{a} \tilde{C}_{\Phi}+\left(C_{\Phi}+C_{a}\right) \tilde{C}_{\Theta_{a}}\right]+n_{\Phi, a}, \\
& n_{\Phi, a}=n_{\Phi}-n_{\Phi_{(a)}},
\end{aligned}
$$

so that (3.7)-(3.9) can be restated as

$$
\Phi_{(a)} \in \mathcal{F}_{C_{\Phi}+C_{a}, \tilde{C}_{\Phi}+\tilde{C}_{\Theta a}(\bmod \mathbf{Z} / \kappa)}^{n_{\Phi}-n_{\Phi, a}} .
$$

The fields $\Theta_{a}$ have spin

$$
s_{\Theta_{a}}=-\alpha_{a a}+n_{\Theta_{a}},
$$

and belong to the parafermionic sector whenever $\alpha_{a a}$ is non-integer. The asymptotic relation between the matrix elements of the energy-momentum tensor and those of parafermionic fields was first noted in [9] on the basis of quantum group arguments.

For each particle $A_{a}$ in the theory a further decomposition of the space of fields according to the asymptotic behavior (3.6) of the form factors is obtained in the form

$$
\mathcal{F}_{C, \tilde{C}}^{n}=\bigoplus_{n_{a} \in \mathbf{Z}} \mathcal{F}_{C, \tilde{C}}^{n, n_{a}}
$$

where $n_{a}$ is the integer in (3.3). In many theories the subspaces $\mathcal{F}_{C, \tilde{C}}^{n, n_{a}}$ turn out to be finitedimensional.

We now investigate under which conditions a spinless field is mapped into another spinless field by the action of the operator $\Lambda_{a}$. We call $\hat{\Omega}_{a, 0}$ the field subspace containing the fields with such a property:

$$
\hat{\Omega}_{a, 0}=\left\{\Phi \mid s_{\Phi}=s_{\Phi(a)}=0\right\} .
$$

Consider $\Phi \in \hat{\Omega}_{a, 0}$. Since $s_{\Phi}=0, \Phi$ can only be a linear combination of spinless fields $\Phi_{i}$ with $C_{\Phi_{i}}$ and/or $\tilde{C}_{\Phi_{i}}$ equal zero, and with $n_{\Phi_{i}}=0$. Similarly, $s_{\Phi_{i(a)}}=0$ implies $y_{\Phi_{i}, a}=n_{\Phi_{i}, a}=0$. At this point, (3.20) with $\Phi=\Phi_{i}$ gives $\left(C_{\Phi_{i}}+C_{a}\right) \tilde{C}_{\Theta_{a}}=0$ if $\tilde{C}_{\Phi_{i}}=0$, and $\left(\tilde{C}_{\Phi_{i}}+\tilde{C}_{\Theta_{a}}\right) C_{a}=0$ if $C_{\Phi_{i}}=0$. Hence, using $C_{\bar{a}}=-C_{a}, \tilde{C}_{\Theta_{\bar{a}}}=-\tilde{C}_{\Theta_{a}}$, and recalling (3.22), we have the three cases 2

$$
\hat{\Omega}_{a, 0}= \begin{cases}\mathcal{F}_{-C_{a}, 0}^{0,0} \oplus \mathcal{F}_{0,-\tilde{C}_{\Theta_{a}}, 0}^{0,0}, & \text { if } C_{a} \neq 0, \tilde{C}_{\Theta_{a}} \neq 0 \\ \bigoplus_{C} \mathcal{F}_{C, 0}^{0,0}, & \text { if } C_{a} \neq 0, \tilde{C}_{\Theta_{a}}=0 \\ \bigoplus_{C} \mathcal{F}_{C, 0}^{0,0} \bigoplus_{\tilde{C}} \mathcal{F}_{0, \tilde{C}}^{0,0}, & \text { if } C_{a}=\tilde{C}_{\Theta_{a}}=0 .\end{cases}
$$

\footnotetext{
${ }^{2}$ Notice that (3.18) implies $\tilde{C}_{\Theta_{a}}=0$ if $C_{a}=0$, unless $C_{b}=0 \forall b$; but if all particles are neutral there is no internal symmetry, and no dual charge. Then the case $C_{a}=0, \tilde{C}_{\Theta_{a}} \neq 0$ is excluded.
} 
The subspace $\hat{\Omega}_{a, 0}$ contains in particular the spinless fields $\phi_{a}$ that $\Lambda_{a}$ maps onto themselves, possibly up to conjugation of $C$ and $\tilde{C}$. It follows from (3.26) and (3.22) that these are the solutions of the equation

$$
\Lambda_{a} \phi_{a}=\lambda_{\phi_{a}} \phi_{\bar{a}}
$$

with $\lambda_{\phi_{a}}$ constant. Denoting $\Omega_{a, 0}$ the subspace of $\hat{\Omega}_{a, 0}$ spanned by the solutions of (3.27), we have

$$
\Omega_{a, 0} \subseteq \begin{cases}\mathcal{F}_{-C_{a}, 0}^{0,0} \oplus \mathcal{F}_{0,-\tilde{C}_{\Theta a}}^{0,0}, & \text { if } C_{a} \neq 0 \\ \bigoplus_{C} \mathcal{F}_{C, 0}^{0,0} \bigoplus_{\tilde{C}} \mathcal{F}_{0, \tilde{C}}^{0,0}, & \text { if } C_{a}=0 .\end{cases}
$$

Notice that, if $C_{a} \neq 0, \phi_{a}$ necessarily has both a charged $(C \neq 0)$ and a neutral $(C=0)$ part,

$$
\phi_{a}=\phi_{a}^{(c)}+\phi_{a}^{(n)}, \quad \text { if } C_{a} \neq 0 .
$$

In particular, we have $F_{a}^{\phi_{a}} \neq 0$, a condition which holds also for $C_{a}=0$, unless $\phi_{a}$ is an order field. Equation (3.12) yields

$$
\lambda_{\phi_{a}}=\frac{F_{a}^{\phi_{a}}}{\left\langle\phi_{\bar{a}}\right\rangle}, \quad \text { if } \quad F_{a}^{\phi_{a}} \neq 0
$$

for the constant in (3.27). Hence, for example, (3.27) written for a particle-antiparticle state gives

$$
\lim _{\theta_{2} \rightarrow+\infty} F_{\bar{a} a}^{\phi_{a}}\left(\theta_{1}, \theta_{2}\right)=\frac{F_{a}^{\phi_{a}} F_{\bar{a}}^{\phi_{\bar{a}}}}{\left\langle\phi_{\bar{a}}\right\rangle}, \quad \text { if } F_{a}^{\phi_{a}} \neq 0
$$

which becomes

$$
\lim _{\theta_{2} \rightarrow+\infty} F_{\bar{a} a}^{\phi_{a}^{(n)}}\left(\theta_{1}, \theta_{2}\right)=\frac{F_{a}^{\phi_{a}^{(c)}} F_{\bar{a}}^{\phi_{\bar{a}}^{(c)}}}{\left\langle\phi_{\bar{a}}^{(n)}\right\rangle}, \quad \text { if } C_{a} \neq 0 .
$$

We will see in the next section that equation (3.27) normally admits solutions, so that $\Omega_{a, 0}$ is non-empty and contains fields whose form factors have the mildest asymptotic behavior which (3.13) allows for spinless fields $\left(y_{\Phi, a}=0\right)$. In this sense the fields $\phi_{a}$ can be called "fundamental", meaning that for any positive $k$ there exist spinless fields $\Phi$ with the same charge and dual charge content as $\phi_{a}$, whose matrix elements are, for this reason, solutions of exactly the same form factor equations, with a different asymptotic behavior, $y_{\Phi, a}=n_{\Phi, a}=k$. We denote $\Omega_{a, k}$ the space spanned by such fields and use the notation

$$
\Omega_{a}=\bigoplus_{k=0}^{\infty} \Omega_{a, k}
$$

for the whole sector of the space of fields related, in the way we just explained, to the fundamental fields $\phi_{a}$. The derivatives $(\partial \bar{\partial})^{k} \phi_{a}$, whose form factors are immediately obtained from those of $\phi_{a}$, are examples of fields belonging to $\Omega_{a, k}$. We know that the action of $\Lambda_{a}$ on a field in $\Omega_{a, k}$ gives a field with spin $-k$.

The structure of $\Omega_{a}$, with its internal gradation in terms of a non-negative integer $k$, is clearly reminiscent of the characterization of the space of fields in conformal field theory [1]. This is not 
totally unexpected once we consider that, up to symmetry breaking effects, the field content of the massive theory and that of its massless (conformal) limit should coincide. Let us recall that in a two-dimensional conformal field theory the space of fields is the direct sum of "families" corresponding to lowest weight representations of the infinite-dimensional Virasoro algebra. All the fields within a family behave in the same way under the internal symmetries of the theory, namely, for the cases of interest in this paper, have the same charge and dual charge. Each family consists of a "primary" field and infinitely many "descendants" whose scaling dimension differs from that of the primary by a positive integer. Restricting our attention to spinless primaries and spinless descendants, the scaling dimension of a primary can be denoted $2 \Delta$ and that of a descendant $2(\Delta+l)$, where $l$ is called level of the descendant; there exist descendants for all positive values of $l$. If we denote by $V_{\Delta, l}$ the space of spinless descendants of level $l$, the restriction to spinless fields of a family associated to a spinless primary can be written as

$$
V_{\Delta}=\bigoplus_{l=0}^{\infty} V_{\Delta, l} .
$$

Considering that the derivative $(\partial \bar{\partial})^{l}$ of the primary belongs to $V_{\Delta, l}$, the hypothesis that $k$ in (3.33) and $l$ in (3.34) play the same role is very natural. We refrain however from a straightforward identification between $k$ and $l$. If it is reasonable to expect that

$$
\Omega_{a}=\bigoplus_{\Delta \in \mathcal{D}_{a}} V_{\Delta}
$$

with the sum taken over the families having the same charge and dual charge content as $\phi_{a} 3$, the hypothesis that $\bigoplus_{\Delta \in \mathcal{D}_{a}} V_{\Delta, l}$ coincides with $\Omega_{a, l}$ is probably too strong in the general case. Indeed, it is difficult to exclude that, for some $\Delta \in \mathcal{D}_{a}, V_{\Delta, l} \subseteq \Omega_{a, k}$ with a fixed positive value of $k-l$.

Even with this caveat, the space $\Omega_{a, 0}$ should be spanned by primary field 4 . On the other hand, by definition, a basis in $\Omega_{a, 0}$ is provided by the solutions of equation (3.27). Whether, when $\operatorname{dim} \Omega_{a, 0}>1$, the basis of solutions of (3.27) coincides with the basis of primary fields, is an essential question. Also, when $\phi_{a}$ splits into two components as in (3.29), the simplest expectation is that these components correspond to primary fields with the same scaling dimension related by a symmetry transformation.

In the next section we illustrate how this scenario appears to be supported by the results so far available for several models. Before that, let us recall that, for reflection positive theories 5 , it was shown in [10] that

$$
y_{\Phi, a} \leq \Delta_{\Phi} \quad \text { for } \quad s_{\Phi}=0,
$$

${ }^{3}$ Consistence of this statement requires

$$
\Omega_{a}=\Omega_{b} \quad \text { if } \quad C_{a}=C_{b} .
$$

\footnotetext{
${ }^{4}$ In the massive context we call primary field a field which becomes a conformal primary in the massless limit.

${ }^{5}$ Reflection positivity requires that the two-point correlation function of any field other than the identity is positive and monotonically decreasing with distance. In particular, it excludes negative scaling dimensions.
} 
where $2 \Delta_{\Phi}$ is the scaling dimension of $\Phi$. Relevant (in the renormalization group sense) fields have $\Delta_{\Phi}<1$, and in a reflection positive theory are necessarily primaries. In practice (3.37) turns out to be extremely constraining for the purpose of the identification of these fields. The lower bound (3.13) helps understanding this circumstance on general grounds.

\section{Application to models}

\section{1 $\mathrm{Z}_{N}$ models}

For an internal symmetry group $G=\mathbf{Z}_{N}, N \geq 2$, the simplest (minimal) solution of the equations (2.5) $-(2.8)$ is a scattering theory [11] of particles $A_{a}, a=1, \ldots, N-1$, with masses

$$
m_{a}=2 m \sin \frac{\pi a}{N},
$$

$\mathbf{Z}_{N}$ charges $C_{a}=a$, and scattering amplitudes

$$
\begin{aligned}
& S_{a b}^{c d}(\theta)=\delta_{a}^{c} \delta_{b}^{d} S_{a b}(\theta) \\
& S_{a b}(\theta)=\prod_{l=0}^{a-1} \prod_{m=0}^{b-1} f_{2 / N}\left(\theta+\frac{i \pi}{N}(a-2 l)-\frac{i \pi}{N}(b-2 m)\right), \\
& f_{\alpha}(\theta)=\frac{\sinh \frac{1}{2}(\theta+i \pi \alpha)}{\sinh \frac{1}{2}(\theta-i \pi \alpha)} .
\end{aligned}
$$

Since $f_{\alpha}(\theta) \rightarrow e^{ \pm i \pi \alpha}$ as $\theta \rightarrow \pm \infty$, the asymptotic phases obtained from (2.10) are

$$
\alpha_{a b}=\frac{a b}{N} .
$$

Chosing $\kappa=-1 / N$ and comparing with (3.18) we obtain

$$
\tilde{C}_{\Theta_{a}}=C_{a}=a .
$$

The fact that $\kappa \tilde{C}$ is defined modulo integers means that $\tilde{C}$ is identified modulo $N$, so it is the charge associated to a group that we can denote $\tilde{Z}_{N}$. As a consequence, the sum in (2.22) runs over integer values from 0 to $N-1$ for both $C$ and $\tilde{C}$. The fields $\Theta_{a}$ with spin

$$
s_{\Theta_{a}}=-\frac{a^{2}}{N}+n_{\Theta_{a}},
$$

are parafermions belonging to $\mathcal{F}_{a, a}$, and appear in the operator product expansion of the order fields in $\mathcal{F}_{a, 0}$ with the disorder fields in $\mathcal{F}_{0, a}$.

These conclusions about the space of fields associated to the minimal $Z_{N}$-invariant scattering theories fully agree with the known fact that these theories describe the scaling limit of $Z_{N^{-}}$ invariant lattice models [8, 12, and are obtained as the symmetry preserving perturbations of the $\mathbf{Z}_{N}$-invariant conformal field theories with central charge $2(N-1) /(N+2)$ constructed in [8]. These theories indeed exhibit a space of fields classified in terms of $Z_{N} \times \tilde{Z}_{N}$ charges. In particular, the spin of the fields in the parafermionic sector is known to be $\pm a^{2} / N$ plus integers, 
and includes (4.7). The "order parameters" $\sigma_{a}, a=1, \ldots, N-1$ (which in our notation are the most relevant fields in $\left.\mathcal{F}_{a, 0}^{0}\right)$ have scaling dimensions $a(N-a) / N(N+2)$, as well as the "disorder parameters" $\mu_{a}$ (the most relevant fields in $\mathcal{F}_{0, a}^{0}$ ). Since the fields $\sigma_{a}$ and $\mu_{a}$ are exchanged by duality trasformations, their relative normalization is fixed by the condition

$$
\lim _{|x| \rightarrow 0} \frac{\left\langle\sigma_{a}(x) \sigma_{N-a}(0)\right\rangle}{\left\langle\mu_{a}(x) \mu_{N-a}(0)\right\rangle}=1 .
$$

Following the reasonings of the previous section, the expectation is that in this case the solutions of (3.27) decompose as in (3.29) with

$$
\phi_{a}^{(c)}=\sigma_{N-a}, \quad \phi_{a}^{(n)}=b_{a} \mu_{N-a},
$$

with $b_{a}$ a constant.

Form factor solutions have been studied in [13, 7] for $N=2$ (Ising model) and in [14, 15] for $N=3$ (three-state Potts model). It can be checked from these results that a solution to (3.27) exists and is unique (up to normalization) for a given $a$; moreover (4.9) holds and $\left|\lambda_{\phi_{a}}\right|=\left|F_{a}^{\sigma_{N-a}} / b_{N-a}\left\langle\mu_{a}\right\rangle\right|$ equals 1 for $N=2, a=1$, and 0.9839 .. for $N=3, a=1,2$.

In principle, the normalization constants $b_{a}$ can be determined exactly through a resummation of the form factor spectral decompositions of the correlators entering (4.8). In practice, this can be achieved only for $N=2$ and gives $\left|b_{1}\right|=1$ (see e.g. [7]). For $N=3$ the result $\left|b_{1}\right|=\left|b_{2}\right|=1$ was obtained within $1 \%$ accuracy in [16] through comparison with high precision Monte Carlo data for the lattice model.

It would be interesting to generalize the analysis to $N>3$ exploiting the form factor formulae obtained in [17, 18].

\subsection{Sine-Gordon model}

The sine-Gordon model is the integrable quantum field theory defined by the action

$$
\mathcal{A}_{S G}=\int d^{2} x\left(\frac{1}{2}\left(\partial_{\mu} \varphi\right)^{2}-\tau \cos \beta \varphi\right) .
$$

The elementary excitations are the solitons $A_{+}$and anti-solitons $A_{-}$which interpolate between adjacent degenerate minima of the periodic potential. Hence, the particles $A_{a}, a= \pm 1$, carry a topologic charge $a$.

It is well known [19] that the sine-Gordon model is equivalent through fermionization to the massive Thirring model, the theory of a Dirac fermion with four-spin interaction which explicitly exhibits invariance (hidden in (4.10)) under a group $G=U(1)$. The solitons of the bosonic action are the Thirring fermions, and the topologic charge is the $U(1)$ charge: $C_{a}=a$. 
The scattering of solitons and antisolitons is determined by the amplitudes [2]

$$
\begin{aligned}
S_{++}^{++}(\theta)=S_{--}^{--}(\theta) & =S_{0}(\theta)=-\exp \left\{-i \int_{0}^{\infty} \frac{d x}{x} \frac{\sinh \frac{x}{2}\left(1-\frac{\xi}{\pi}\right)}{\sinh \frac{x \xi}{2 \pi} \cosh \frac{x}{2}} \sin \frac{\theta x}{\pi}\right\}, \\
S_{+-}^{+-}(\theta)=S_{-+}^{-+}(\theta) & =-\frac{\sinh \frac{\pi \theta}{\xi}}{\sinh \frac{\pi}{\xi}(\theta-i \pi)} S_{0}(\theta), \\
S_{+-}^{-+}(\theta)=S_{-+}^{+-}(\theta) & =-\frac{\sinh \frac{i \pi^{2}}{\xi}}{\sinh \frac{\pi}{\xi}(\theta-i \pi)} S_{0}(\theta), \\
\xi & =\frac{\pi \beta^{2}}{8 \pi-\beta^{2}} .
\end{aligned}
$$

The asymptotic diagonality condition (2.10) is satisfied with

$$
\alpha_{a b}=-\frac{1}{4}\left(1+\frac{\pi}{\xi}\right) a b=-\frac{2 \pi}{\beta^{2}} a b, \quad a, b= \pm 1 .
$$

Comparison with (3.18) (with the choice $\kappa=1$ ) and (3.23) gives

$$
\begin{aligned}
& \tilde{C}_{\Theta_{a}}=\frac{2 \pi}{\beta^{2}} a(\bmod \mathbf{Z}), \\
& s_{\Theta_{a}}=\frac{2 \pi}{\beta^{2}}+n_{\Theta_{a}} .
\end{aligned}
$$

Since $\beta$ is a continuous parameter the dual charge is not quantized, and the sum in (2.22) runs over all integers for $C$, and over the interval $[0,1)$ for $\tilde{C}$.

The charges $C$ and $\tilde{C}$ can indeed be identified for the primary fields of the sine-Gordon model. These can be written as (see e.g. [20])

$$
U_{m, \nu}(x)=e^{i\left[\frac{2 \pi}{\beta} m \tilde{\varphi}(x)+\nu \beta \varphi(x)\right]},
$$

where $m \in \mathbf{Z}$ is the $U(1)$ charge. At the gaussian fixed point the bosonic field can be written as $\varphi(x)=\phi(z)+\bar{\phi}(\bar{z})$, and $\tilde{\varphi}(x) \equiv \phi(z)-\bar{\phi}(\bar{z})$. The fields (4.18) have semi-locality indices and spin given by

$$
\begin{aligned}
& \gamma_{\left(m_{1}, \nu_{1}\right),\left(m_{2}, \nu_{2}\right)}=m_{1} \nu_{2}+m_{2} \nu_{1}, \\
& s_{m, \nu}=m \nu .
\end{aligned}
$$

Recalling (2.23) and (2.24), we have

$$
C_{U_{m, \nu}}=m, \quad \tilde{C}_{U_{m, \nu}}=\nu(\bmod \mathbf{Z}) .
$$

The fields $U_{m, 0}$ and $U_{0, \nu}$ are spinless and have scaling dimensions [20] $\pi m^{2} / \beta^{2}$ and $\nu^{2} \beta^{2} / 4 \pi$, respectively. In particular, the trace of the energy-momentum tensor $\Theta \sim \cos \beta \varphi \sim U_{0,1}+U_{0,-1}$ belongs to $\mathcal{F}_{0,0}^{0}$, as it should, and has scaling dimension $\beta^{2} / 4 \pi$.

From the first line of (3.28) we expect that the solutions of (3.27) are

$$
\phi_{ \pm}=U_{\mp 1,0}+b_{ \pm} U_{0, \mp 2 \pi / \beta^{2}}=e^{\mp i \frac{2 \pi}{\beta} \tilde{\varphi}}+b_{ \pm} e^{\mp i \frac{2 \pi}{\beta} \varphi}
$$


with $b_{ \pm}$constants. Observe that the fields in the linear combination, which we select on these grounds, have indeed the same scaling dimension $\pi / \beta^{2}$. Moreover, if the results for the form factors of the fields (4.18) given in [21, 22] are not sufficient to check that (4.22) satisfy (3.27), one can check that $U_{0,-2 \pi a / \beta^{2}}$ indeed belongs to $\mathcal{F}_{0,-2 \pi a / \beta^{2}}^{0, n_{a}}$ with $n_{a}=0$.

For $\beta^{2}<4 \pi$ the soliton-antisoliton interaction is attractive and the amplitudes (4.12), (4.13) possess simple poles at $\theta=i(\pi-n \xi)$ corresponding to bound states (breathers) $B_{n}$ with masses

$$
m_{n}=2 M \sin \frac{n \xi}{2}, \quad 1 \leq n<\left[\frac{\pi}{\xi}\right]
$$

where $M$ is the soliton mass and $[x]$ denotes the integer part of $x$. The lightest breather $B_{1}$ is the particle interpolated by the boson $\varphi$. Since $C_{B_{n}}=0$, we also have $\tilde{C}_{\Theta_{B_{n}}}=06$. In this case the space $\Omega_{B_{n}, 0}$ of solution of the equation

$$
\Lambda_{B_{n}} \phi_{B_{n}}=\lambda_{\phi_{B_{n}}} \phi_{B_{n}}
$$

is expected to contain all the fields (4.18) with zero spin (i.e. with $m$ or $\nu$ equal zero). The form factor results of [23, 21, 24] indicate that the fields $U_{0, \alpha}$ satisfy (4.24) and that

$$
\lambda_{\left(U_{0, \alpha}\right)_{B_{1}}}=-2 i \cos \frac{\xi}{2} \sqrt{2 \sin \frac{\xi}{2}} \exp \left[-\int_{0}^{\xi} \frac{d t}{2 \pi} \frac{t}{\sin t}\right] \frac{\sin \xi \alpha}{\sin \xi} .
$$

\subsection{Theories without internal symmetries}

In an integrable theory without internal symmetries all particles are neutral, there is no mass degeneracy and the scattering is necessarily diagonal. Hence (2.10) is satisfied and $\alpha_{a b}=0 \forall a, b$, as implied by (3.18) and $C_{a}=0 \forall a$. This agrees with the known fact that in these cases all the amplitudes can be expressed as

$$
\begin{gathered}
S_{a b}(\theta)=\prod_{\gamma \in \mathcal{G}_{a b}} t_{\gamma}(\theta), \\
t_{\gamma}(\theta)=\frac{\tanh \frac{1}{2}(\theta+i \pi \gamma)}{\tanh \frac{1}{2}(\theta-i \pi \gamma)} .
\end{gathered}
$$

Since $C_{\Phi}=\tilde{C}_{\Phi}=0 \forall \Phi$, it follows from (2.24) that all the fields have integer spin and that (2.22) and (2.25) reduce to the sum

$$
\mathcal{F}=\bigoplus_{s \in \mathbf{Z}} \mathcal{F}_{0,0}^{s}
$$

over subspaces with spin $s$. Such subspaces are further decomposed as in (3.24) according to the value of $n_{\Phi, a}=y_{\Phi, a}$. The solutions of (3.27) all belong to $\mathcal{F}_{0,0}^{0,0}$ and have $\lambda_{\phi_{a}}=F_{a}^{\phi_{a}} /\left\langle\phi_{a}\right\rangle$. Equation (3.35) now becomes

$$
\Omega_{a}=\mathcal{F}_{0,0}^{0}=\bigoplus_{\Delta} V_{\Delta}
$$

\footnotetext{
${ }^{6}$ One can check that the soliton-breather and breather-breather scattering amplitudes [2] go asymptotically to 1 .
} 
where the value of $a$ is immaterial, as required by (3.36) , and the sum runs over all values of $\Delta$ allowed in the theory. For this kind of theories, equation (3.27) amounts to a particular case of the asymptotic factorization property for form factors proposed in [25] and generalized in [26].

A particularly interesting example within this class of theories is the scaling Ising model with magnetic field at critical temperature [27]. The $S$-matrix bootstrap has initial condition (4.26) with $\mathcal{G}_{11}=\{2 / 3,2 / 5,1 / 15\}$ and closes on eight species of particles $A_{a}, a=1, \ldots, 8$, with different masses. Results for the form factors in this theory have been obtained in [10, 28, 29] and are reviewed in [30]. The evidence is that equation (3.27) admits two solutions, the same for all values of $a$, in agreement with (3.36) and with the fact that the Ising model possesses two non-trivial (i.e. other than the identity) primary fields, the spin $\sigma$ and the energy $\varepsilon$. In particular, for $a=1$ one obtains

$$
\lambda_{\phi_{1}}=\left\{\begin{array}{l}
-0.640902 . . \\
-3.70658 . .
\end{array}\right.
$$

The form factor solution corresponding to the field $\sigma$ can be easily identified because in this model $\sigma \sim \Theta$, and yields a ratio $F_{1}^{\sigma} /\langle\sigma\rangle$ which coincides with the upper value in (4.30). On the other hand, numerical estimates on the lattice model give $\left|F_{1}^{\sigma} /\langle\sigma\rangle\right|=0.6408(3)$ and $\left|F_{1}^{\varepsilon} /\langle\varepsilon\rangle\right|=$ 3.707(7) [31], providing substantial evidence that (3.27) correctly selects also the second primary field.

Infinite series of integrable theories without internal symmetries are obtained from massive perturbations of the non-reflection-positive conformal minimal models $\mathcal{M}_{2,2 m+3}, m=1,2, \ldots$, with central charge $1-3(2 m+1)^{2} /(2 m+3)$. The case $m=1$ corresponds to the Lee-Yang model describing the edge singularity of the zeros of the partition function of the Ising model in an imaginary magnetic field [32]-[36]. The form factor results of [37] for $m=1$ and [38] for $m=2,3$ indicate that the above mentioned asymptotic factorization property, and consequently equation (3.27), admit $m$ solutions, in perfect agreement with the number of non-trivial primary fields present at criticality.

For the Lee-Yang model the isomorphism between the conformal space of fields and that determined by the form factor equations has been shown in [6, 39]. It gives, in particular,

$$
\Omega_{a, l}=\mathcal{F}_{0,0}^{0, l}=\bigoplus_{\Delta} V_{\Delta, l},
$$

with the sum running over the two values allowed in the theory, $\Delta=0$ (the identity family 7 ) and $\Delta=-1 / 5$. This shows that for the Lee-Yang model the identification between $k$ in (3.33) and $l$ in (3.34) is complete.

\footnotetext{
${ }^{7}$ Since the identity cannot be considered as a solution of (3.27), this trivial primary should be added to the l.h.s. of (4.31) for $l=0$.
} 


\section{Conclusion}

In this paper we identified a number of general facts concerning the classification of quantum fields in integrable theories with asymptotically diagonal scattering and additive charges. An essential role is played by the asymptotic behavior of form factors, which allows for the introduction of operators $\Lambda_{a}$ mapping fields into fields. A notion of massive primary field is naturally associated to these operators through equation (3.27). There is evidence for several models that the fields selected in this way become conformal primaries in the massless limit. The possibility of classifying scalar fields into subspaces labelled by a non-negative integer indicates that the level structure implied by conformal symmetry at fixed points is recovered starting from particle dynamics away from criticality.

According to (3.36), operators $\Lambda_{a}$ associated to particles with the same charge are expected to select the same sector of the space of fields. Generically, however, not all values of charge are realized by single-particle states. For example, the $\mathbf{Z}_{N}$ models do not possess neutral particles, while in sine-Gordon there are no particles with charge larger than 1 in absolute value. It seems reasonable to expect that for these cases the corresponding sectors of field space are associated to operators of the form $\Lambda_{a_{1}} \ldots \Lambda_{a_{n}}$, with $\sum_{i=1}^{n} C_{a_{i}}$ equal to the desired charge value. So, as an example, the field $\Theta$ in the $\mathbf{Z}_{N}$ models would be solution of the equation $\Lambda_{a} \Lambda_{\bar{a}} \phi=\lambda \phi$.

The generalization of our analysis to integrable theories outside the class we considered here is also an interesting issue 8 . It seems plausible that equations of type (3.11) can serve as a definition for operators $\Lambda_{a}$ in more general integrable theories, or even in absence of integrability. Speculations about higher dimensions are also possible.

Acknowledgments. Work supported in part by the ESF grant INSTANS.

\footnotetext{
${ }^{8}$ See [40] for results on the asymptotic properties of form factors in the $O(n)$ non-linear sigma model with $n>2$.
} 


\section{References}

[1] A.A. Belavin, A.M. Polyakov and A.B. Zamolodchikov, Nucl. Phys. B 241 (1984) 333.

[2] A.B. Zamolodchikov and Al.B. Zamolodchikov, Ann. Phys. 120 (1979) 253.

[3] M. Karowski, P. Weisz, Nucl. Phys. B 139 (1978) 455.

[4] F.A. Smirnov, Form Factors in Completely Integrable Models of Quantum Field Theory, World Scientific, 1992. Phys. A 3 (1988) 743.

[5] J.L. Cardy and G. Mussardo, Nucl. Phys. B 340 (1990) 387.

[6] G. Delfino and G. Niccoli, Nucl. Phys. B 799 (2008) 364.

[7] V.P. Yurov and Al.B. Zamolodchikov, Int. J. Mod. Phys. A6 (1991) 3419.

[8] A.B. Zamolodchikov and V.A. Fateev, Sov. Phys. JETP 62 (1985) 215; Sov. Phys. JETP $63(1986) 913$.

[9] F.A. Smirnov, Comm. Math. Phys. 132 (1990) 415.

[10] G. Delfino and G. Mussardo, Nucl. Phys. B 455 (1995) 724.

[11] R. Koberle and J.A. Swieca, Phys. Lett. B 86 (1979) 209.

[12] G.F. Andrews, R.J. Baxter and P.J. Forrester, J. Stat. Phys. 35 (1984) 193.

[13] B. Berg, M. Karowski and P. Weisz, Phys. Rev. D 19 (1979) 2477.

[14] A.N. Kirillov and F.A. Smirnov, Kiev preprint ITF-88- 73R, 1988 (in russian).

[15] G. Delfino and J. Cardy, Nucl. Phys. B 519 (1998) 551.

[16] M. Caselle, G. Delfino, P. Grinza, O. Jahn and N. Magnoli, J. Stat. Mech. 0603:P008, 2006.

[17] H. Babujian, A. Foerster and M. Karowski, Nucl. Phys. B 736 (2006) 169.

[18] V.A. Fateev, V.V. Postnikov and Y.P. Pugai, JETP Lett. 83 (2006) 172.

[19] S. Coleman, Phys. Rev. D 11 (1975) 2088.

[20] A.B. Zamolodchikov and Al.B. Zamolodchikov, Sov. Sci. Rev. A. Phys. 10 (1989) 269-433.

[21] S. Lukyanov, Mod.Phys.Lett. A 12 (1997) 2543.

[22] S. Lukyanov and A.B. Zamolodchikov, Nucl. Phys.B 607 (2001) 437.

[23] A. Koubek and G. Mussardo, Phys. Lett. B 311 (1993) 193.

[24] S. Lukyanov, Phys. Lett. B 408 (1997) 192. 
[25] G. Delfino, P. Simonetti and J.L. Cardy, Phys. Lett. B 387 (1996) 327.

[26] G. Delfino and G. Niccoli, Nucl. Phys. B 707 (2005) 381.

[27] A.B. Zamolodchikov, Advanced Studies in Pure Mathematics 19 (1989) 641; ; Int. J. Mod. Phys. A 3 (1988) 743.

[28] G. Delfino and P. Simonetti, Phys. Lett. B 383 (1996) 450.

[29] G. Delfino, P. Grinza and G. Mussardo, Nucl. Phys. B B 737 (2006) 291.

[30] G. Delfino, J. Phys. A 37 (2004) R45.

[31] M. Caselle and M. Hasenbusch, Nucl. Phys. B 579 (2000) 667.

[32] C.N. Yang and T.D. Lee, Phys. Rev. 87 (1952) 404.

[33] T.D. Lee and C.N. Yang, Phys. Rev. 87 (1952) 410.

[34] M.E. Fisher, Phys. Rev. Lett. 40 (1978) 1610.

[35] J.L. Cardy, Phys. Rev. Lett. 54 (1985) 1354.

[36] J.L. Cardy and G. Mussardo, Phys. Lett. B 225 (1989) 275.

[37] Al. B. Zamolodchikov, Nucl. Phys. B 348 (1991) 619.

[38] C. Acerbi, G. Mussardo and A. Valleriani, J. Phys. A 30 (1997) 2895.

[39] G. Delfino and G. Niccoli, J. Stat. Mech. (2005) P04004.

[40] J. Balog and P. Weisz, Nucl. Phys. B 778 (2007) 259. 